\title{
A Study of Fluctuations in Sun's Radiation by Measuring Earth's Angular Velocity
}

Hossieni $H^{*}$, Jabar MAF, Burhan M, Abdulqadr IH and Zhiar M. Jaza

Department of Physics, School of Science, University of Sulaimani, Iraq

\begin{abstract}
During a normal day, the intensity of sunlight, as a vector, is measured. By calculating variation of angle between sun's ray and surface of the earth, angular velocity of the Earth's rotation at different time intervals is determined. This angular velocity is not unchanging and traces back to the variations in intensity of light emanating from the Sun.
\end{abstract}

Keywords: Angular velocity; Earth; Rotation; Luminosity; Fluctuation; Sun; Radiation

\section{Introduction}

Students learn from physics textbooks on Sun and solar system that the Sun is an ebullient oven which via nuclear fusion produces light and heat. This radiation provides heat to our planet and the solar system [1]. We know that producing light and heat is not an unchanging process but is both completely non-linear and unpredictable [2].

Our planet, Earth, spins or rotates about its axis and at the same time rotates around the Sun, as other planets in the solar system do. The rotation around the Sun is on an elliptical path. One can easily calculate period of the angular velocity of the Earth's rotation by measuring lengths of a day and a year and then determine period of revolution around the Sun. The period of rotation of the Earth is about 23 hours, 56 minutes and 4.09 seconds, and one can calculate angular velocity by using this well-known relation:

$$
\omega=\frac{2 \pi}{T}
$$

Where $\mathrm{T}$ is the period of rotation and $\pi \approx 3.14$. Therefore, angular velocity of the Earth $(\omega)$ can be calculated and it turns out to be $7.29 \times$ $10^{-5} \mathrm{rad} / \mathrm{s}$ [2]. It is obvious that the Sun rises from east and sets in the west. The intensity of its light starts to build up from early morning, reaching maximum at noon, then begins to decrease in strength as day goes by. Evidently, the angle of incidence of sunlight and the earth's surface changes during the day. In a cloudless dust-free day, one can measure the change in this angle by measuring the variations in the sunlight intensity. This can be done through using:

$$
\omega=\frac{\Delta \theta}{\Delta T}
$$

Where $\Delta \theta$ is the change in the sun's angle of incidence and $\Delta t$ is the time of this change. Equations (1) and (2) are usually expected to produce same results. But this is not so as the intensity of the sunlight and it is not constant. We can, in each time interval, observe a difference between semi-theoretical and full experimental result. We have, in each time interval, a different angular velocity. This can be traced back to variations in sunlight due to its perpetual internal nuclear activities.

In this research, we present our measurements of the intensity of the sunlight in Sulaimani city having coordinates $\left(35^{\circ} 33^{\prime} 26^{\prime \prime} \mathrm{N} 45^{\circ} 26^{\prime} 08^{\prime \prime} \mathrm{E}\right)$. These are performed on January the 6th 2015 using a normal Lux-meter (Figure 1). We also present how to use our measurements to deduce the variations in the Sun's corona.

\section{Results and Discussion}

We conducted our measurements on the variations of the intensity of sunlight in the city of Sulaimani on 6th of January of 2015.Then these collected data were used to calculate the angular velocity of the Erath. The day was a clear, dust-free cloudless one which was ideal for our measurements. It was expected then that any change in the radiation with the time of the day is attributed to the change of angle between sun's rays and earth's axis. Figure 2 shows the variation of sun's radiation, taken hourly, and the curve clearly peaks at 12:30 which indicates recording of maximum radiation. At this point, the sunlight makes $0^{\circ}$ angle with earth's axis. As we descend from the peak, the recorded radiation decreases as expected. Let's represent the luminosity at any time by a vector ( I) and that at noon by $\mathrm{I} 0$ (vectors 2 and 1 in Figure 3 respectively). The luminosity's vector (I) makes an angle $\theta$ with 1 . And $\theta$ can be calculated using the following equation:

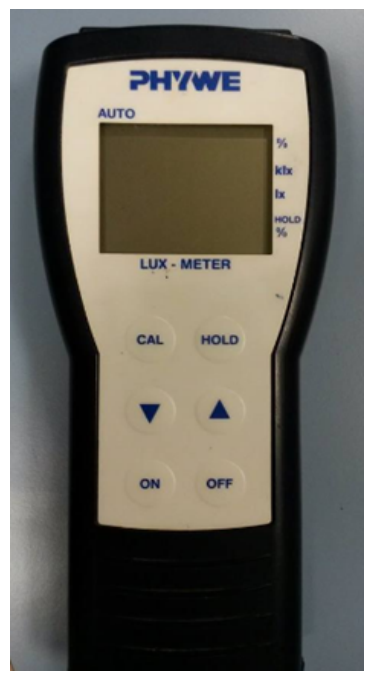

Figure 1: Lux-Meter.

*Corresponding author: Hossieni $\mathrm{H}$, Department of Physics, School of Science, University of Sulaimani, Iraq. Tel: +964-770-1991949; E-mail: hossien.hossieni@univsul.edu.iq

Received December 07, 2015; Accepted January 02, 2016; Published January 12, 2016

Citation: Hossieni H, Jabar MAF, Burhan M, Abdulqadr IH, Jaza ZM (2016) A Study of Fluctuations in Sun's Radiation by Measuring Earth's Angular Velocity. J Climatol Weather Forecasting 4: 151. doi:10.4172/2332-2594.1000151

Copyright: @ 2016 Hossieni H, et al. This is an open-access article distributed under the terms of the Creative Commons Attribution License, which permits unrestricted use, distribution, and reproduction in any medium, provided the original author and source are credited. 


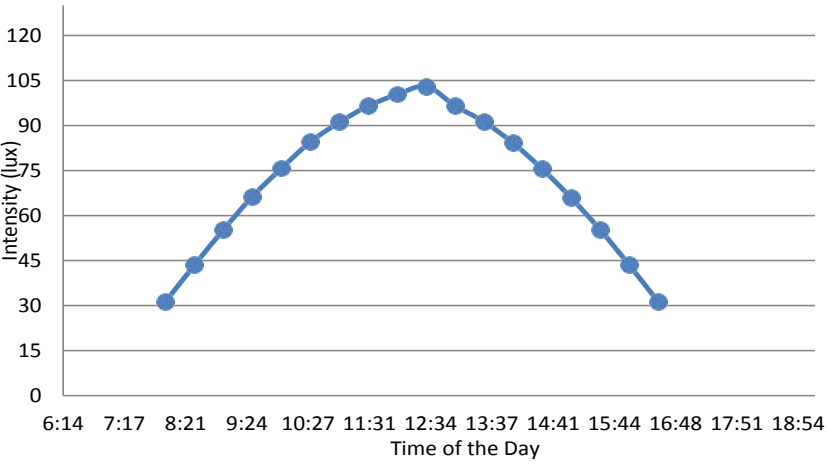

Figure 2: Variation of sunlight intensity with time from 08:00 to 16.30

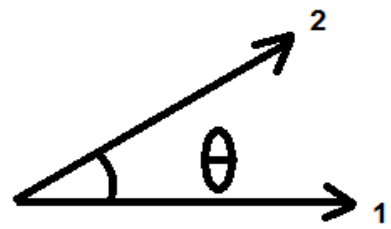

Figure 3: 1 represents intensity vector at noon (maximum value) while 2 is intensity vector at any other time.

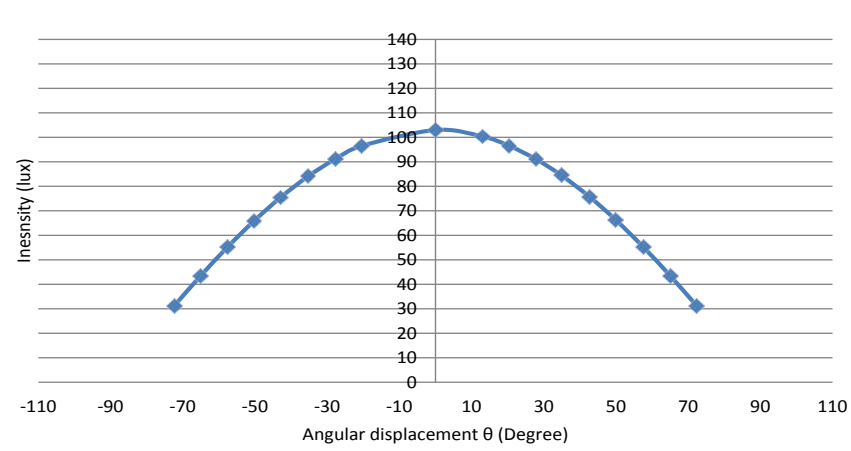

Figure 4: Variation of sunlight intensity with angle of incidence between Sun's ray and earth axis.

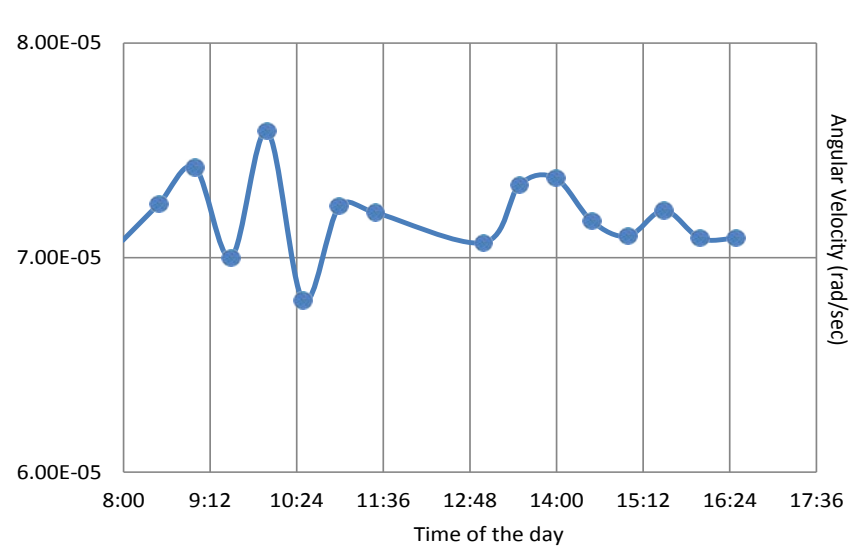

Figure 5: Variation of measured angular velocity with time.

$$
\theta=\cos ^{-1} \frac{I}{I_{0}}
$$

Figure 4 shows the variation of luminosity with angle on 6th January 2015 and it is clear that the trend of our data follows a Gaussian distribution. We started our observation from 08:00 to 16:30 and these correspond to angles from $-72.3^{\circ}$ to $+72^{\circ}$. We mentioned earlier that the angular velocity of Earth is the ratio of changes in angle to that of time. This ratio should remain the same if the rate of Sun's radiation is invariable. But Figure 5 shows noticeably that the measured angular velocity of the earth varies with time. This angular velocity fluctuates between $-7 \%$ to $+4 \% \mathrm{Rad} / \mathrm{s}$ from the standard value.

The angular velocity of Earth is not constant due to the annual fluctuations in the earth's gravitational constant. This and Chandler period, the drift of geomagnetic field, cause earthquakes, fluctuations in the circulation of the atmosphere and other geophysical phenomena $[4,5]$. In consideration of the large scale of the solar system, all of the Earth's related fluctuations can be ignored. The variation of angular velocity of earth, according to our measurements, relates to the change in the luminosity of the sunlight. We know the radiation of the sun cannot be constant and when we calculate angular velocity of the earth by measuring the Sun's luminosity this error creeps in our calculations [6]. We can relate the majority of these fluctuations to the variation in the intensity of light coming from sun's corona.

\section{References}

1. Genesis mission (2002) A Public Outreach Module: Sunlight and Solar Heat: How Are They Made?

2. Miyamoto K (1980) Plasma physics for nuclear fusion, Massachusetts Institute of Technology Cambridge, MA: 625

3. World book Inc (1984) World Book Encyclopedia Vol. 6 Illinois Scott Fetzer Company.

4. Munk WH, Miller RL (1950) Variation in the Earth's Angular Velocity Resulting from Fluctuations in Atmospheric and Oceanic Circulation 3: 94-99.

5. Kropotkina N, Trapeznikov YA (1965) Variation of the angular velocity of the Earth's rotation, the oscillation of the pole and the rate of drift of the geomagnetic field and their possible association with geotectonic processes, International Geology Review 7: 1949-1962.

6. NASA (2009) New Sun-Watching Instrument to Monitor Sunlight Fluctuations
Citation: Hossieni H, Jabar MAF, Burhan M, Abdulqadr IH, Jaza ZM (2016) A Study of Fluctuations in Sun's Radiation by Measuring Earth's Angular Velocity. J Climatol Weather Forecasting 4: 151. doi:10.4172/2332-2594.1000151 